\title{
Systematic Analysis of the Lysine Succinylome in Candida albicans
}

Hailin zheng ${ }^{1,2}$, Yun He ${ }^{1}$, Xiaowei Zhou ${ }^{1,2}$, Guanyu Qian ${ }^{1}$, Guixia Lv ${ }^{1}$, Yongnian Shen ${ }^{1}$, Jiyun Liu ${ }^{3}$, Dongmei $\mathrm{Li}^{1,4}$, Xiaofang $\mathrm{Li}^{1,2^{*}}$, Weida Liu ${ }^{1,2^{*}}$

1Department of Medical Mycology, Institute of Dermatology, Chinese Academy of

Medical Science and Peking Union Medical College, Nanjing 210042, Jiangsu,

People's Republic of China; 2Jiangsu Key Laboratory of Molecular Biology for Skin

Diseases and STIs, Nanjing 210042, Jiangsu, People's Republic of China; 3Jingjie

PTM Biolab (Hangzhou) Co.,Ltd., Hangzhou 310018, Zhejiang, People’s Republic of

China;4 Georgetown University Medical Center, Department of Microbiology \&

Immunology, Washington, DC, 20057,USA.

Tables of supporting information

\begin{tabular}{|l|l|}
\hline & Main content \\
\hline Figure S1 & $\begin{array}{l}\text { Western blot analysis of whole cell lysate using succinyl-lysine } \\
\text { antibody demonstrate the presence of succinylated proteins }\end{array}$ \\
\hline Figure S2 & $\begin{array}{l}\text { Venn diagram show the overlap among 5 parallel enrichments of } \\
\text { total protein solution }\end{array}$ \\
\hline Figure S3 & $\begin{array}{l}\text { Representative MS/MS spectra of succinylpeptides from protein } \\
\text { ACO (P82611) }\end{array}$ \\
\hline Figure S4 & Assignment of secondary structure types in C. albicans \\
\hline Table S1 & Protein and functional annotation \\
\hline Table S2 & Comparison with other species \\
\hline
\end{tabular}


Figure S1

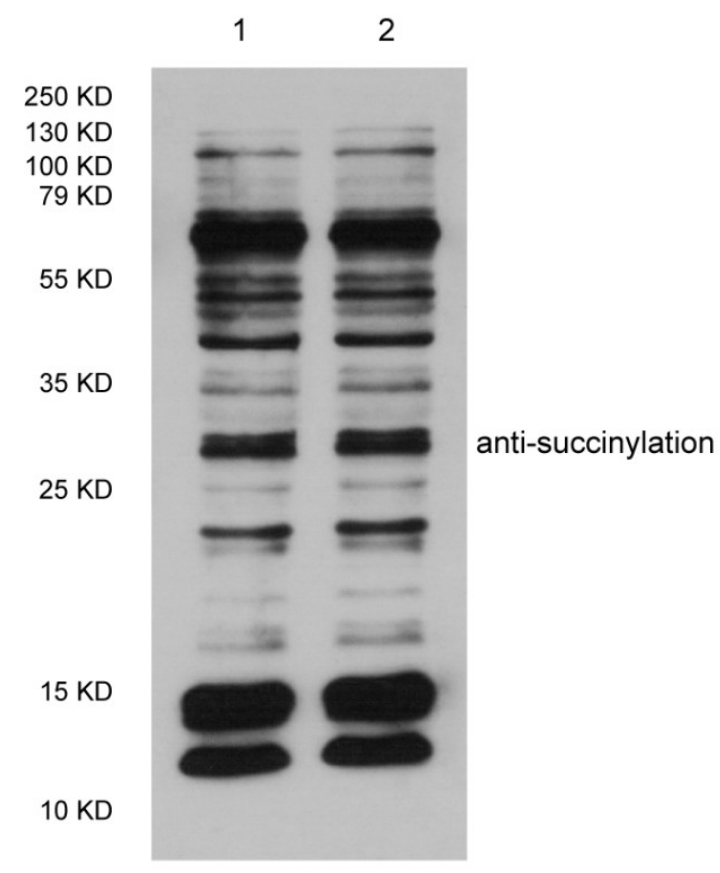

Figure S1. Western blot analysis of whole cell lysate using succinyl-lysine antibody demonstrate the presence of succinylated proteins. Lane1 and lane2 were two parallel Western blot experiments. 
Figure S2

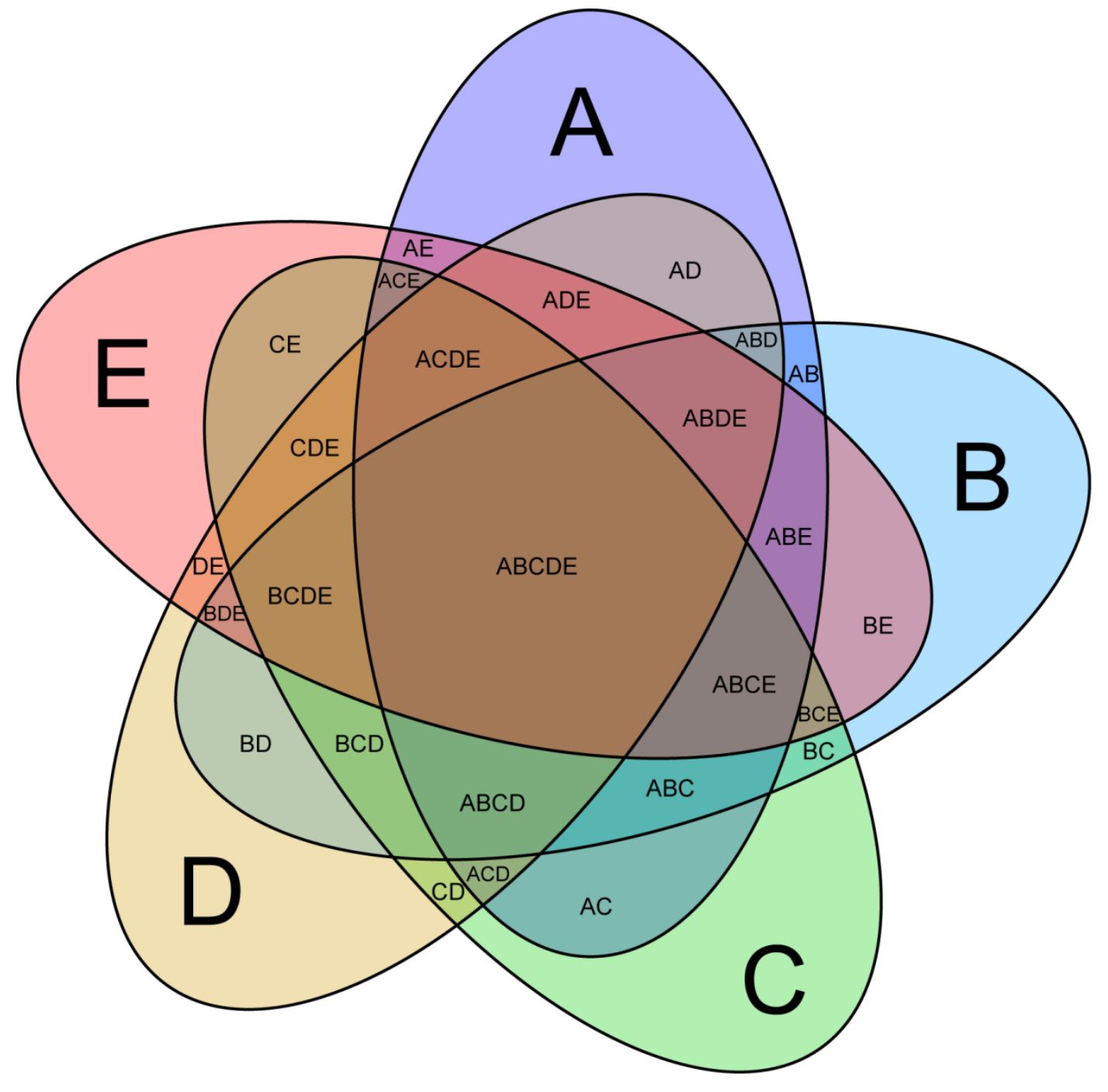

Figure S2. Venn diagram show the overlap among 5 parallel enrichments of total protein solution . 
Figure S3

A

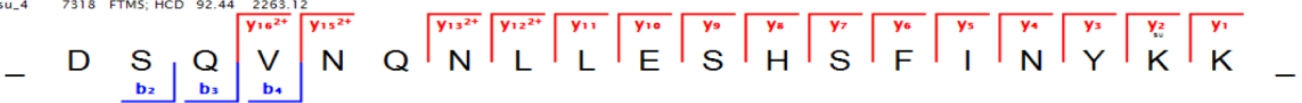

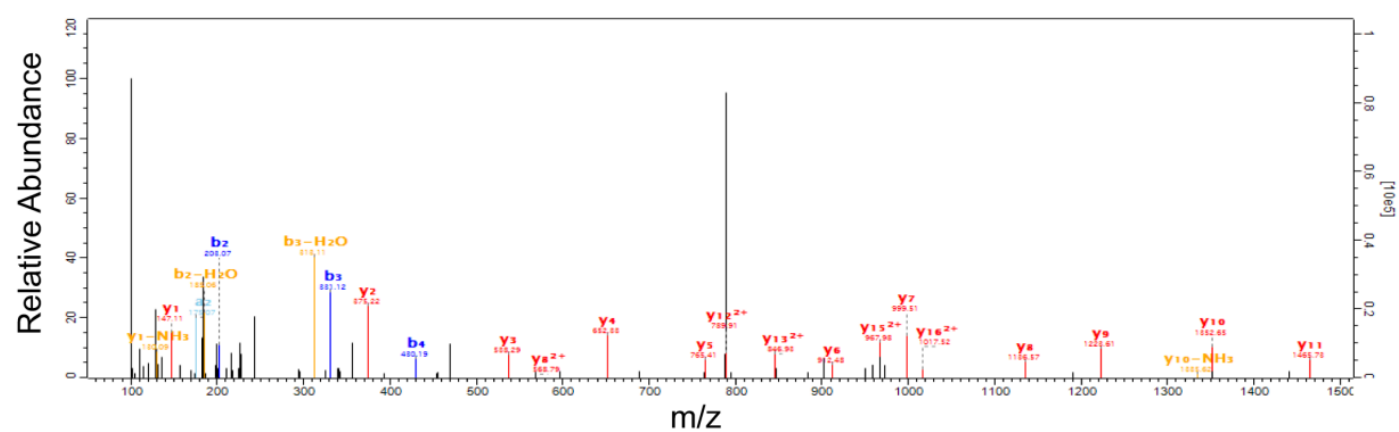

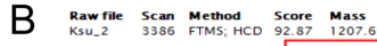
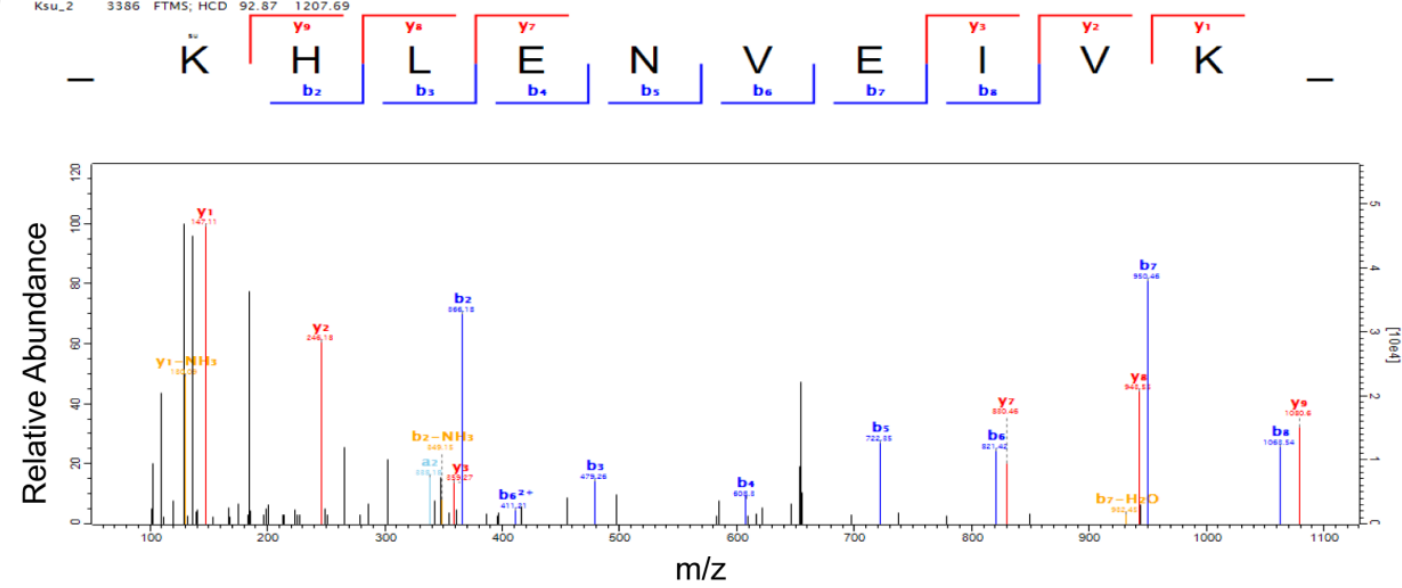

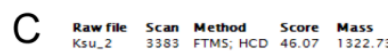
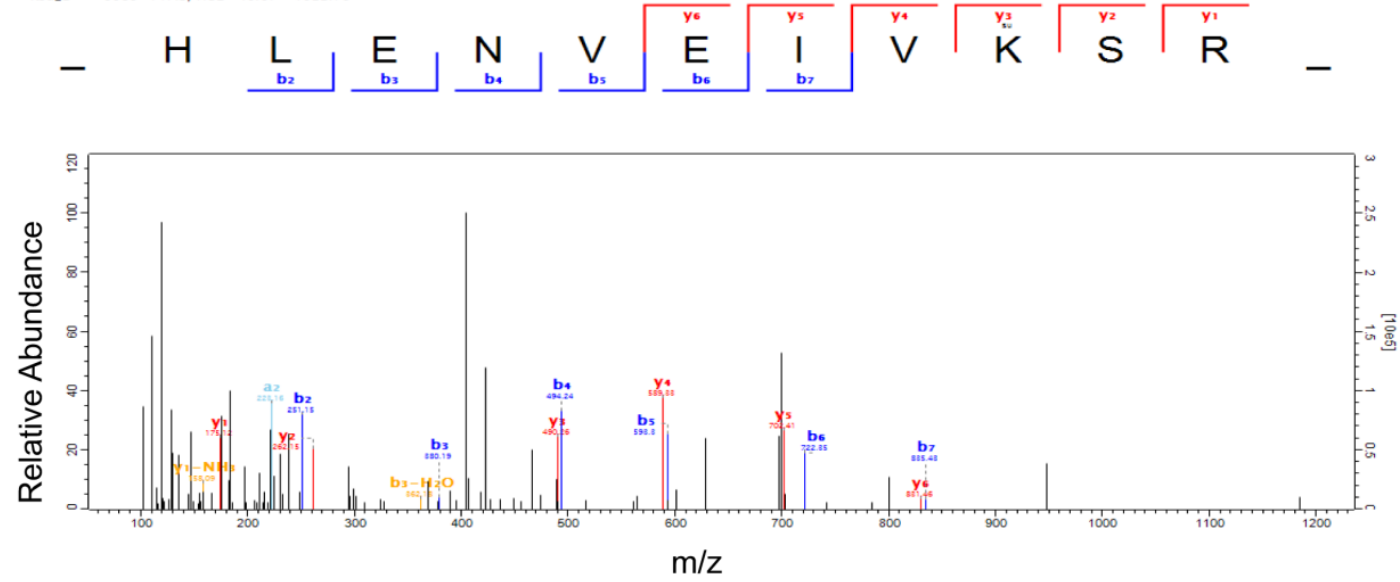

Figure S3. Representative MS/MS spectra of succinylpeptides from protein ACO (P82611): (A) succinylpeptide _DSQVNQNLLESHSFINYK(su)K_ with an succinylation site at K43, (B) succinylpeptide _K(su)HLENVEIVK_ with an succinylation site at K44, (C) succinylpeptide _ HLENVEIVK(su)SR _ with an succinylation site at K53. 


\section{Figure S4}

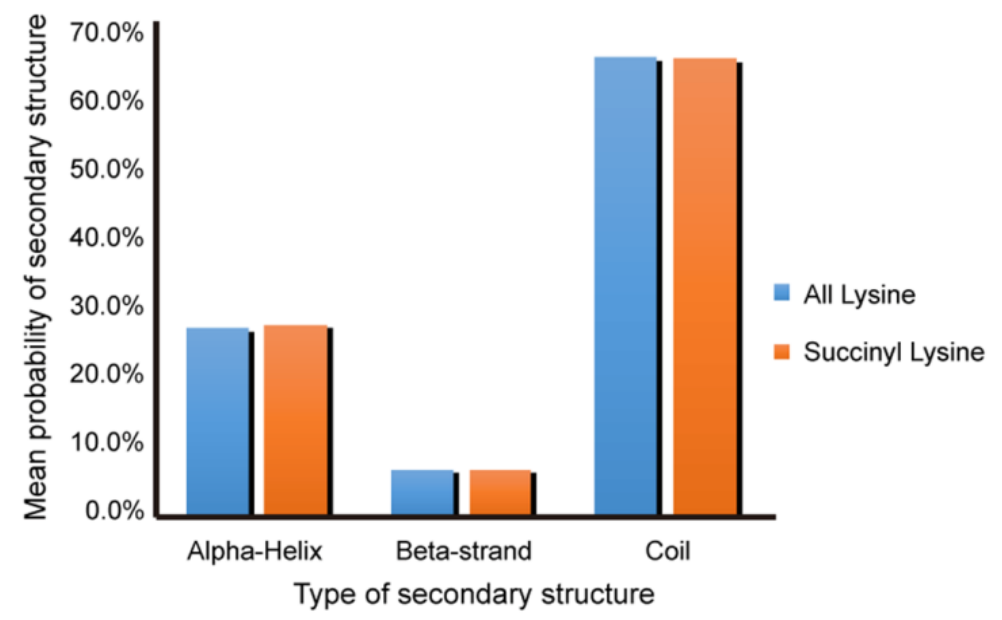

Figure S4. Assignment of secondary structure types in C. albicans. Percentage of all lysines and succinylated lysines within secondary structure types separately. 